\title{
Portal Vein Thrombosis Secondary to Occult Polycythemia Vera
}

\author{
Antón FR Gameiro, António Robalo Nunes, Paula Guerra, Estela Mateus, Fátima Fernandes \\ Hospital do SAMS, Lisbon, Portugal
}

Doi: 10.12890/2020_002003 - European Journal of Case Reports in Internal Medicine - C EFIM 2020

Received: $15 / 09 / 2020$

Accepted: 01/10/2020

Published: 02/11/2020

How to cite this article: Gameiro AFR, Robalo Nunes A, Guerra P, Mateus E, Fernandes F. Portal vein thrombosis secondary to occult polycytemia vera. EJCRIM 2020;7: doi:10.12890/2020_002003.

Conflicts of Interests: The Authors declare that there are no competing interests.

This article is licensed under a Commons Attribution Non-Commercial 4.0 License

\section{ABSTRACT}

Portal vein thrombosis (PVT) is an uncommon finding in patients without cirrhosis. The underlying $x \backslash$ aetiology is challenging and the condition has a wide differential diagnosis. We present a case of PVT in an anaemic patient with chronic iron and folic acid deficiency masking underlying polycythemia vera (PV). Only a careful review of the patient's clinical history allowed the identification of a short period of laboratory erythrocytosis, 6 months before the clinical onset of PVT, while the patient was on iron and folic acid supplementation. The finding raised clinical suspicion of PV previously masked by iron deficiency anaemia. Subsequent investigation confirmed the presence of the JAK2 V617F mutation and, ultimately, showed that the patient met all diagnostic criteria for PV. Myeloproliferative disorders (MPD) are associated with systemic prothrombotic states. PV is distinguished clinically from other MPD by the presence of increased red blood cell mass. Moreover, patients with abnormal haematocrit values in the pre-JAK2 V617F era may have had occult or latent PV. Diagnosis confirmation requires a combination of major and minor criteria to capture occasional cases of occult PV. This case emphasizes the importance of always considering MPD in the aetiological investigation of PVT, even in patients who apparently do not fulfil the diagnostic criteria.

\section{LEARNING POINTS}

- We describe a rare gastroenterological presentation of a haematological condition, which provided an unexpected diagnosis.

- Myeloproliferative disorders should always be considered in the investigation of portal vein thrombosis.

\section{KEYWORDS}

Ascites, portal vein thrombosis, polycythemia vera, anaemia, iron deficiency

\section{CASE DESCRIPTION}

She had been previously diagnosed with chronic anaemia due to iron and folic acid deficiency, associated with haemorrhoid bleeding and Hashimoto's thyroiditis. There was no history of alcohol intake and her family history was irrelevant. From an obstetric point of view, she was G2P2.

Initial investigation with abdominal Doppler-US showed portal vein thrombosis (PVT) with cavernous transformation (Fig. 1) and normal liver echogenicity, confirmed by a CT scan and MRI. Superior mesenteric vein thrombosis was also revealed, together with marked ascites and confirmation of normal liver parenchyma structure.

Blood tests showed mild normocytic anaemia, with haemoglobin $11.3 \mathrm{~g} / \mathrm{dl}$, haematocrit 34.5\%, MCV 90.3 fl, MCH 29.6 pg, RDW 19.8\% and platelets $419 \times 103 / \mu \mathrm{l}$, with no alterations in leukocytes. These findings were compatible with absolute iron deficiency and mild folic acid deficiency: serum iron $53 \mu \mathrm{g} / \mathrm{dl}$, transferrin $289 \mu \mathrm{g} / \mathrm{dl}$, ferritin $17 \mathrm{ng} / \mathrm{ml}$ and folic acid $1.4 \mathrm{ng} / \mathrm{ml}$. Hepato-biliary and renal function were normal. 
In light of these findings, the initial diagnostic approach was directed towards hypercoagulable states, and neoplastic and autoimmune diseases, other than thyroiditis. Antibodies against cardiolipin, $\beta$-2-glycoprotein and lupus anticoagulant were negative, ruling out antiphospholipid syndrome. Also, anti-nuclear antibodies and anti-dsDNA were negative, making systemic lupus erythematosus improbable. Normal levels of protein S, protein C and anti-thrombin were found. The prothrombin G20210A mutation was also excluded.

Thyroid ultrasound, and cranial, thoracic, abdominal and pelvic CT scans were performed, with negative results for solid tumours. Mammography excluded breast malignancy. Colonoscopy and oesophagogastroduodenoscopy showed clinically irrelevant findings.

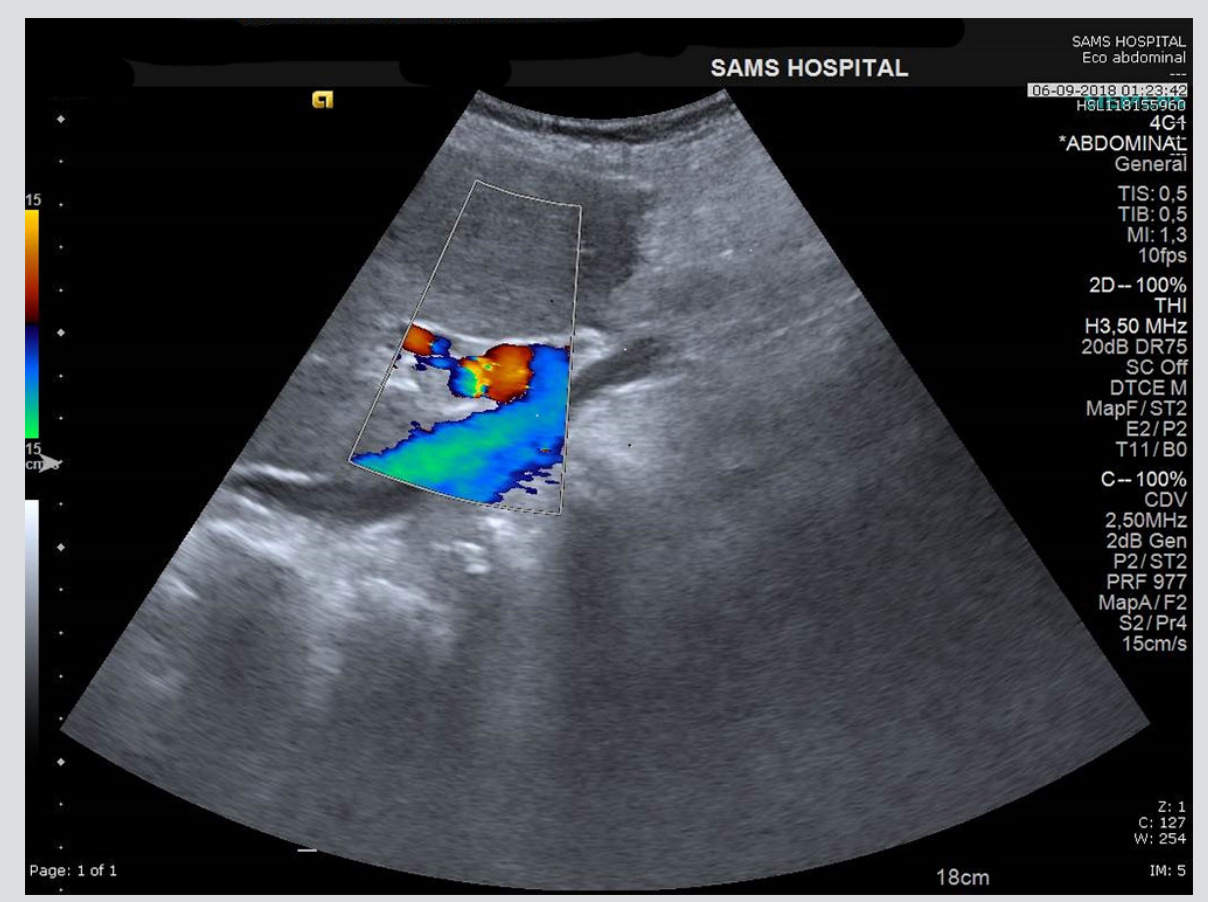

Figure 1. Portal vein thrombosis

However, careful review of the patient's previous clinical history found a short period of laboratory erythrocytosis 6 months before PVT clinical onset. Between 2012 and 2018, the patient's haemoglobin concentrations ranged from $8.1 \mathrm{~g} / \mathrm{dl}$ to $13.0 \mathrm{~g} / \mathrm{dl}$. Iron status indicators at their lowest levels were: iron $19 \mu \mathrm{g} / \mathrm{dl}$, transferrin saturation $4 \%$ and ferritin $7.6 \mathrm{ng} / \mathrm{l}$. At the beginning of 2018 , oral iron and folic acid supplementation was initiated and haemoglobin levels had risen to $16.7 \mathrm{~g} / \mathrm{dl}$ and haematocrit to 52.3\% by April 2018.

Following these findings, testing for the JAK2 V617F mutation was ordered and was positive. Low levels of erythropoietin (EPO) were also found. Bone marrow biopsy showed a hypercellular bone marrow with trilineage growth (Fig. 2) and megakaryocyte aggregation (Figs. 3 and Fig. 4), which is in line with polycythemia vera (PV) diagnostic criteria, based on the WHO classification ${ }^{[1]}$. The patient is currently on lowdose aspirin and anticoagulation therapy with recanalization of the superior mesenteric vein, preserving cavernous transformation in the portal vein, as expected, after 3 months. Hydroxyurea was added as the patient has a high-risk of thrombosis recurrence ${ }^{[2]}$.

\section{DISCUSSION}

PVT is an uncommon finding in the absence of cirrhosis ${ }^{[3]}$. The underlying aetiology in this condition has a wide differential diagnosis, including hypercoagulable states, and autoimmune or myeloproliferative disorders (MPD). Myeloproliferative neoplasms are associated with systemic prothrombotic states and are an uncommon cause of PVT ${ }^{[2,3]}$. PV is distinguished clinically from other MPD by the presence of an increased red blood cell mass. This myeloid malignancy is characterized by a JAK2 (Janus kinase 2; 9p24) mutation, 96\% of which are in exon 14 (JAK2 V617F) and an increased likelihood of developing thrombotic complications ${ }^{[2]}$.

PV diagnostic criteria were recently revised in the WHO Classification of Tumours of Haematopoietic and Lymphoid Tissues. Detection of the JAK2 V617F mutation is highly sensitive (97\% sensitivity) for PV and very nearly $100 \%$ specific in the presence of increased haematocrit. The possibility of a false laboratory result is effectively addressed by measuring serum EPO level, which is below normal in over $85 \%$ of patients with $\mathrm{PV}{ }^{[2]}$. 


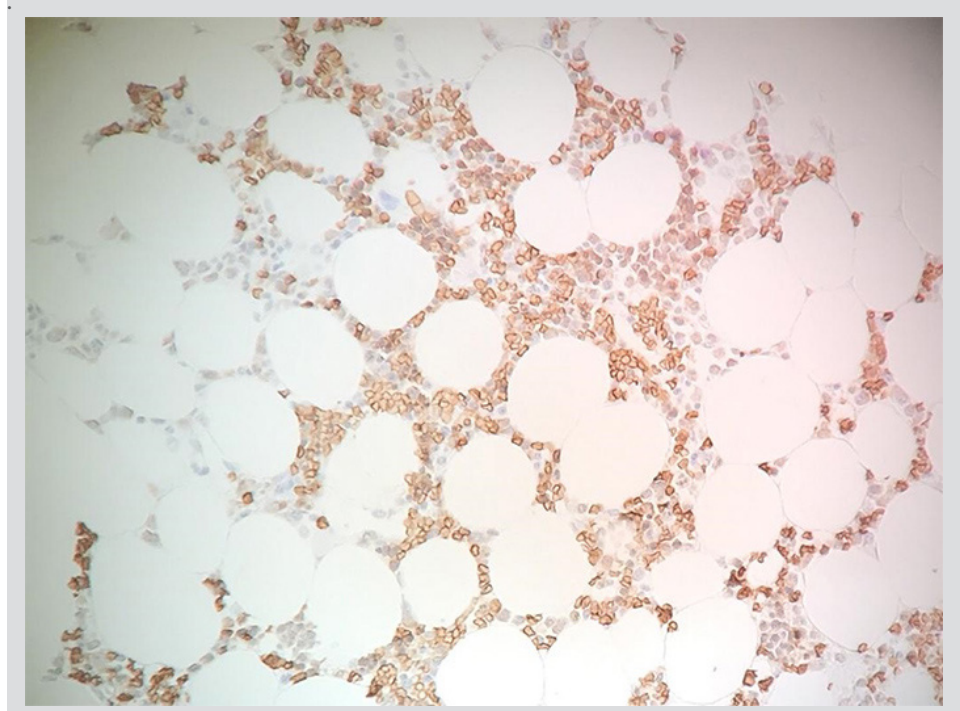

Figure 2. Erythroid precursor hyperplasia (immunochemistry, glycophorin A)

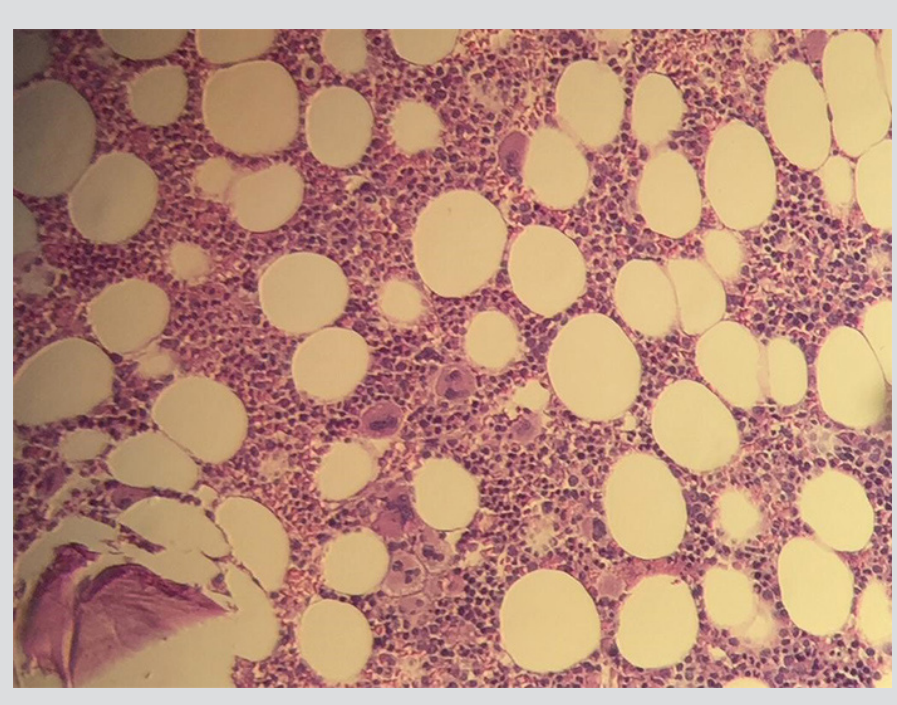

Figure 3. Megakaryocyte aggregation (haematoxylin-eosin $\times 10$ )

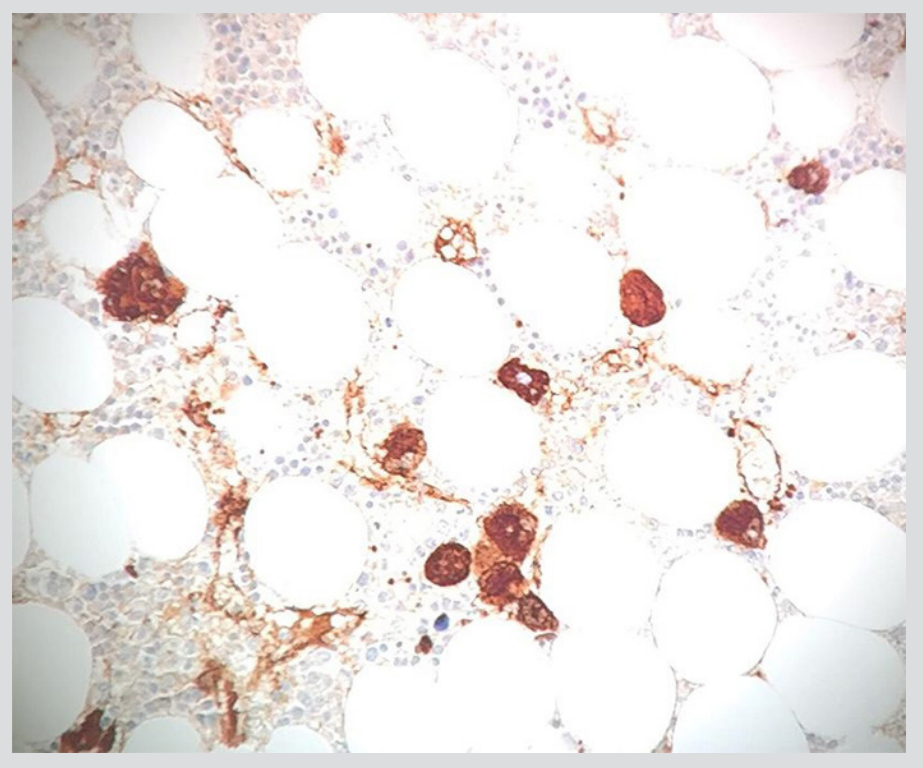

Figure 4. Megakaryocyte aggregation (immunohistochemistry, factor VIII)

The vascular complications in PV due to increased blood viscosity include microvascular circulatory findings such as erythromelalgia, peripheral ischaemia, myocardial infarction, atypical cerebral ischaemic attacks, and major arterial and venous thrombotic events ${ }^{[1]}$.

Median survival for PV patients in general is 14 years and 24 years for those below 60 years of age. The leukaemic transformation rate at 20 years is estimated to be $<10 \%$ for PV. Risk factors reducing survival include advanced age, venous thrombosis and abnormal karyotype ${ }^{[2]}$.

This was a complicated case demonstrating an uncommon onset of a masked pre-existing condition. The presentation with ascites and anaemia did not initially raise suspicion of PV. Chronic iron and folic acid deficiency for long time hid underlying PV, which was only suspected after a thrombotic event.

Bleeding haemorrhoids were initially considered the cause of blood loss, however iron deficiency is frequently diagnosed in PV patients during the course of their disease. This co-existence of iron deficiency and polycythemia presents a physiological disconnection. Iron metabolism is regulated by hepcidin, depending on circulating iron levels, erythroblast secretion of erythroferrone, and inflammation. Dysregulation of iron metabolism in PV is not fully understood ${ }^{[4]}$.

The prognostic scoring systems used for risk-adapted therapy in PV are based on the likelihood of patients developing thrombotic complications. Two factors, age above 60 years and thrombosis history, confer a high risk of recurrent thrombosis. In addition to low-dose 
aspirin and, if needed, phlebotomy to achieve a haematocrit target of 45\%, high-risk patients with PV should receive hydroxyurea as a firstline cytoreductive drug, in order to minimize their risk of thrombosis. Patients who are either intolerant or resistant to hydroxyurea can be effectively managed with INF- $\alpha$ (pegylated preferred) or busulfan ${ }^{[2]}$.

Recently, Kambali and Taj have also reported a case of PV masked by severe iron deficiency anaemia due to menorrhagia ${ }^{[5]}$. That diagnosis also required review of the patient's previous records.

These cases highlight the importance of taking an accurate clinical history and the fact that anaemia, paradoxically, is a possible finding in PV patients. Also, our report emphasizes the importance of always considering MPD in the aetiological investigation of thrombotic scenarios, even in patients with anaemia who at first do not fulfil PV criteria.

Dentali et al. found JAK2 V617F mutations in 49.0\% of patients with idiopathic splanchnic vein thrombosis (SVT), with or without the presence of MPD at the time of diagnosis. MPD developed subsequently in $52.4 \%$ of SVT patients without initial diagnostic criteria. In contrast, JAK2 V617F mutations were found in only $0.9 \%$ of patients with deep venous thrombosis or pulmonary embolism, in $2.6 \%$ of those with cerebral vein thrombosis, and in $1.0 \%$ of patients with retinal vein occlusion ${ }^{[6]}$.

In light of the high prevalence of JAK2 V617F mutations in SVT patients with unsuspected MPD, screening for these mutations in all patients who present with SVT should be considered in order to facilitate timely recognition of MPD and early initiation of treatment.

\section{REFERENCES}

1. Michiels JJ. Erythromelalgia and vascular complications in polycythemia vera. Semin Thromb Hemost 1997;23:441-454

2. Tefferi A, Barbui T. Polycythemia vera and essential thrombocythemia: 2019 update on diagnosis, risk-stratification and management. Am J Hematol 2019;94(1):133-143.

3. Trebicka J, Strassburg CP. Etiology and complications of portal vein thrombosis. Viszeralmedizin 2014;30:375-380.

4. Ginzburg YZ, Feola M, Zimran E, Varkonyi J, Ganz T, Hoffman R. Dysregulated iron metabolism in polycythemia vera: etiology and consequences. Leukemia 2018;32:21052116.

5. Kambali S, Taj A. Polycythemia vera masked due to severe iron deficiency anemia. Hematol Oncol Stem Cell Ther 2018;11(1):38-40.

6. Dentali F, Squizzato A, Brivio L, Appio L, Campiotti L, Crowther M, et al. JAK2V617F mutation for the early diagnosis of Ph- myeloproliferative neoplasms in patients with venous thromboembolism: a meta-analysis. Blood 2009;113:5617-5623. 